\title{
A queda da desigualdade de renda corrente e a participação do $1 \%$ de domicílios de maior renda, 2000-2010
}

\section{CLAUDIO SALVADORI DEDECCA*}

The reduction of income inequality and the participation of the top $1 \%$ in the Brasil, 2000-2010. According to OECD, the recent process of deterioration of the income distribution chain in developing and developed countries has been marked by increased participation of $1 \%$ of households with higher income. In the past decade, Brazil has escaped the general trend of deterioration of the income distribution. This paper shows that the reduction of economic inequality was accompanying the reluctance of the participation of top $1 \%$, arguing that the reproduction of the movement more generally requires that this participation has been reduced in the current decade, to enable sustained growth and development with social justice.

Keywords: Inequality; income distribution.

JEL Classification: D63.

Este ensaio aborda a evolução da participação do primeiro percentil superior no total da renda corrente dos domicílios brasileiros entre 1991 e 2010. Desde os anos 1990, tem se observado crescente interesse em analisar a participação do primeiro percentil superior, em geral denominado como $1 \%$ tope da distribuição de renda corrente. A maioria das iniciativas é justificada na deterioração da distribuição de renda corrente nos países desenvolvidos a partir da década de 1980, que tem sido marcada por uma redução da participação dos estratos inferiores e intermediários e um incremento daquela relativa do estrato $10 \%$ superior, em especial do $1 \%$ tope da distribuição. Esse movimento recebeu especial atenção tanto da

\footnotetext{
* Professor Titular de Economia Social e Do Trabalho da Universidade Estadual de Campinas, Unicamp. E-mail: Claudio.dedecca@gmail.com. Este ensaio contou com o apoio do CNPq. Submetido: 23/ agosto/2012; Aprovado: 23/julho/2013.
} 
Organização das Nações Unidas (2005) como da Organização de Cooperação de Desenvolvimento Econômico (2008 e 2011) ${ }^{1}$.

$\mathrm{Na}$ última década, quando se analisa a evolução global da distribuição de renda corrente, nota-se que a experiência brasileira apresentou trajetória destoante das observadas tanto nos países desenvolvidos como nos em desenvolvimento. Em documento publicado em 2011, a OCDE ressalta a renitência do processo de deterioração da distribuição de renda corrente, ressaltando como exceção a trajetória brasileira. De acordo com a instituição,

The landmark 2008 OECD report Growing Unequal? showed that the gap between rich and poor had been growing in most OECD countries. Three years down the road, inequality has become a universal concern, among both policy makers and societies at large. Today in advanced economies, the average income of the richest $10 \%$ of the population is about nine times that of the poorest $10 \%$. In some countries such as Israel and the United States - inequality has increased further. But even in traditionally egalitarian countries — such as Germany, Denmark and Sweden - the income gap between rich and poor is expanding - from 5 to 1 in the 1980s to 6 to 1 today. Only a few countries have been able to buck this trend: income inequality has recently fallen in Chile and Mexico, but the richest in these two countries still have incomes more than 25 times those of the poorest. In emerging economies, economic growth has helped to reduce sharply the prevalence of poverty. But at the same time high levels of income inequality have risen further. Among the BRICs, only Brazil managed to reduce inequality substantially, although with a ratio of 50 to 1 it is still a far more unequal country than any of the OECD countries. The economic crisis has added urgency to deal with the policy issues related to inequality (OECD, 2011, p. 17).

Apesar do caráter inegavelmente positivo em termos gerais, cabe perguntar como se comportou a participação do $1 \%$ tope de domicílios no movimento de redução da desigualdade da distribuição de renda corrente. A divulgação do Censo Demográfico de 2010 permite tratar adequadamente o tema, que é o objeto foco deste esforço.

O ensaio está organizado em três seções, além desta introdução e das observações finais. Na primeira são apresentadas algumas informações metodológicas sobre os dados de renda adotados no estudo. Em seguida, apresenta-se uma síntese da tendência de redução da desigualdade de renda corrente no Brasil na década passa-

\footnotetext{
${ }^{1}$ Em razão do grande número de estudos realizados sobre o tema, indica-se a leitura de alguns que podem permitir um mapeamento mais amplo dos esforços realizados, como APSA (2004), Atkinson et al. (2009), Dell (2007), Gustafsson et al. (2007), Landais (2007), Leigh (2009), Moriguchi et al. (2007), Nolan (2007) e Pikkety et al. (2006). A conduta metodológica adotada neste estudo permite comparação dos resultados observados para o Brasil com os encontrados nos diversos estudos mencionados.
} 
da. Finalmente, é analisada a renitência da participação do $1 \%$ tope no processo e a necessidade de alteração dessa situação visando ao crescimento sustentado que possa se traduzir em desenvolvimento com maior justiça social na atual década.

\section{SOBRE O PROCEDIMENTO METODOLÓGICO ADOTADO}

As análises sobre a participação do $1 \%$ tope de domicílios têm sido realizadas a partir das informações produzidas pelos levantamentos domiciliares dos sistemas nacionais de estatística. Os estudos focam fundamentalmente os países desenvolvidos, em razão da maior disponibilidade e da maior qualidade dos dados por eles produzidos. A perspectiva analítica pode ser conduzida para o Brasil a partir dos dados dos censos demográficos, que permitem representatividade para as informações sobre renda do último percentil.

A informação encontrada nesses levantamentos refere-se à renda corrente dos domicílios, isto é, da renda que eles auferem regularmente e que constitui lastro do padrão de consumo que os domicílios realizam cotidianamente. O procedimento adotado nos censos demográficos segue orientação da Organização Internacional do Trabalho, que tem como referência o enfoque metodológico construído por John Hicks $(1940,1948)$. Este enfoque dá também suporte às orientações gerais estabelecidas pelas Nações Unidas para elaboração das Contas Nacionais para seus países-membros (Canberra Group, 2001; Zacharias, 2002). Ele considera que a renda corrente expressa o poder de compra realizado cotidianamente pelas famílias, em uma situação de estabilidade de seu patrimônio (estoque). Se por um lado a adoção da renda corrente estabelece limitações quanto às informações sobre o padrão de vida realizado e potencial dos domicílios, por não incorporar mudanças na renda disponível decorrentes de alterações no patrimônio (Canberra, 2001; Weinberg, 2004, 2006), tem-se, por outro, que a disponibilidade desta informação, com metodologia comum internacionalmente e estável ao longo do tempo, viabiliza em grande medida a realização de análises comparadas entre países e com perspectiva longitudinal, dado que possíveis erros tendem a ser recorrentes no tempo e comuns às metodologias nacionais (Pikkety et al. 2006; Weinberg, 2004, 2006).

A perspectiva metodológica adotada neste ensaio segue as orientações da Organização Internacional do Trabalho (1998 e 2003) para tratamento da informação de renda, incorporando também as encaminhadas no documento do Canberra Group (2001) quanto à complexidade do processo de formação da renda no atual estágio de desenvolvimento do capitalismo. O tratamento da informação estatística e o foco da análise se enquadram na abordagem adotada em estudos de outras experiências nacionais pelos diversos autores mencionados e pela Organização de Cooperação para o Desenvolvimento Econômico (OECD, 2008). Dentre os estudos existentes, cabe destacar os organizados em Atkinson (2009) e o elaborado recentemente por este autor (2013). A adoção da metodologia encontrada nesses estudos para este ensaio tem grande importância, pois a ela possibilita a comparação dos resultados encontrados para o Brasil com aqueles apresentados para outros países. 
Da mesma forma que conduzem os autores internacionais e as instituições multilaterais mencionadas, este estudo procura explorar os resultados de modo simples, dando as principais evidências da participação dos domicílios 1\% tope na distribuição de renda corrente. Os motivos para esta conduta são (a) a pouca disponibilidade de análise desse estrato para a experiência brasileira e (b) os limites da representatividade estatística para um grupo de domicílios relativamente pequeno.

Feitas essas observações gerais sobre as razões da conduta metodológica adotada neste ensaio, são apresentadas algumas observações sobre o procedimento de tratamento dos dados de renda que foi utilizado, o qual é totalmente compatível com as orientações da OIT (1998 e 2003) e do Canberra Group (2001).

Quadro 1: Tipos de Rendimento Monetário e Não Monetário segundo Dimensões Básicas de Desigualdades

\begin{tabular}{|c|c|c|}
\hline Dimensões de Desigualdades & $\begin{array}{l}\text { Formas Básicas de } \\
\text { Rendimento }\end{array}$ & Tipos de Rendimentos \\
\hline \multirow{7}{*}{ Econômica } & \multirow{5}{*}{ Renda Corrente (Fluxo) } & $\begin{array}{l}\text { Renda do Trabalho (salário, } \\
\text { remuneração do trabalho } \\
\text { autônomo, pro labore, renda } \\
\text { da profissão liberal) }\end{array}$ \\
\hline & & Renda da Proteção Social \\
\hline & & Renda de Aluguéis \\
\hline & & Renda de Juros e Dividendos \\
\hline & & $\begin{array}{l}\text { Produção para Próprio } \\
\text { Consumo }\end{array}$ \\
\hline & \multirow{2}{*}{ Patrimônio (Estoque) } & Ativos Imobiliários \\
\hline & & Ativos Financeiros \\
\hline \multirow{11}{*}{ Social } & \multirow{11}{*}{$\begin{array}{c}\text { Em espécie ( Com ou sem } \\
\text { alguma contrapartida } \\
\text { monetária) }\end{array}$} & Água e Saneamento \\
\hline & & Agrária \\
\hline & & Alimentar \\
\hline & & Educação \\
\hline & & Energia \\
\hline & & Habitação \\
\hline & & Meio Ambiente \\
\hline & & Previdência \\
\hline & & Trabalho \\
\hline & & Saúde \\
\hline & & Transporte \\
\hline
\end{tabular}

Fonte: The Canberra Group, Expert Group on Household Income Statistics, Final Report and Recommendation, Canberra. Elaborado pelo autor a partir das recomendações apresentadas no relatório e considerando as determinações da Constituição Federal de 1988.

Considerando as formas de renda monetária e não monetária (Quadro 1), observa-se que a maioria dos levantamentos domiciliares não abrange ou abrange muito parcialmente as rendas oriundas de ganhos de patrimônio, tampouco 
abarca adequadamente as auferidas em espécie. Somente pesquisas de despesa dos domicílios permitem captar parte das rendas não monetárias. Por se ter origem em amostra em geral de tamanho mais reduzido devido ao custo do levantamento, tem-se que a informação para o $1 \%$ tope produzida pelas pesquisas de despesas apresenta maior restrição em termos de desagregação para a análise. Mesmo que de modo restrito à renda corrente, os censos demográficos, por recolherem a renda domiciliar no questionário básico, propiciam um informação com maior possibilidade de desagregação em termos regionais, de estratos ou de segmentos sociais ${ }^{2}$.

Essas observações metodológicas relativas às informações sobre renda nos levantamentos domiciliares são fundamentais para explicitar o escopo analítico dos estudos a partir da distribuição de renda corrente, os quais permitem conhecer uma dimensão relevante da desigualdade socioeconômica, que, entretanto, apresenta configuração mais complexa e abrangente como indicado no Quadro $1^{3}$.

\section{A QUEDA DA DESIGUALDADE DE RENDA CORRENTE NO BRASIL NESTE INÍCIO DE SÉCULO}

Tem sido expressivo o esforço de pesquisadores na análise da evolução recente da desigualdade da distribuição de renda corrente no Brasil. Uma avaliação desse esforço e das principais abordagens adotadas pode ser realizada a partir dos ensaios de Oliveira (2006), Saboia (2007), Barros et al. (2007 e 2008), Hoffmann (2007), Dedecca (2007), Salm (2007), Kertenetzky (2008), Lavinas (2010), Barros et al. (2010), Soares (2011) e Cacciamali (2011). Todas eles reconhecem a redução da desigualdade, bem como analisaram seus determinantes a partir da informação de renda corrente, antes da incidência tributária, que é propiciada pela Pesquisa Nacional de Amostra de Domicílios — PNAD/IBGE. Uma síntese do movimento é apresentada na Tabela 1 .

Os diversos estudos realizados discutem fundamentalmente a maior ou menor contribuição que as rendas do trabalho e da proteção social tiveram para a redução

\footnotetext{
${ }^{2}$ Como será explorado mais à frente, a restrição da análise à renda corrente deve ser considerada como ponderável se o objetivo for conhecer a desigualdade econômica nessa dimensão. O processo de financeirização da riqueza e das rendas, ocorrido nas últimas décadas, tem reduzido a importância do rendimento corrente na renda total dos estratos superiores, como apontam os estudos Pikkety et al. (2006), Weinberg (2004 e 2006) e o levantamento Survey Consumer Finances do Federal Reserve dos Estados Unidos. Mesmo considerando ser a renda disponível do $1 \%$ tope significativamente mais expressiva que a indicada pela renda corrente, se verá neste ensaio que a situação de desigualdade nessa dimensão restrita é substantiva e se manteve renitente ao longo da década anterior.

${ }^{3}$ Em relação ao debate sobre a complexidade da desigualdade socioeconômica, ver Kusnetz (1973), Sem (1992), Osberg et al. (2003), Gadrey et al. (2003), APSA (2004), DESA/UM (2005), Bihr et al. (2008) e Wolff et al. (2009).
} 
da desigualdade de renda corrente ${ }^{4}$. O conjunto dos esforços permitiu mapear e explicitar a contribuição que alguns institutos de política pública, como o salário mínimo e a transferência de renda, e o aumento do emprego tiveram para a evolução da desigualdade. Em resumo, ao se falar da queda desigualdade de renda corrente ocorrida, os estudos abordaram e abordam uma dimensão relevante, mas que é insuficiente para revelar a evolução e a complexidade do quadro de desigualdades socioeconômicas presente no país, seja no que se refere aos determinantes econômicos como aos de natureza social ${ }^{5}$.

Tabela 1: Indicadores de rendimento familiar per capita segundo estratos de renda, Brasil, 2001-2009

\begin{tabular}{|c|c|c|c|c|c|}
\hline & 2001 & 2003 & 2005 & 2007 & 2009 \\
\hline \multicolumn{6}{|c|}{ Índice de evolução do rendimento médio do estrato } \\
\hline $10 \%-$ & 100.0 & 101.2 & 135.3 & 142.8 & 155.5 \\
\hline $20 \%$ & 100.0 & 103.7 & 128.4 & 144.8 & 163.2 \\
\hline $30 \%$ & 100.0 & 101.7 & 120.1 & 137.8 & 156.3 \\
\hline $40 \%$ & 100.0 & 100.1 & 116.4 & 133.7 & 150.7 \\
\hline $50 \%$ & 100.0 & 100.0 & 115.4 & 133.4 & 148.0 \\
\hline $60 \%$ & 100.0 & 101.3 & 113.8 & 131.9 & 145.4 \\
\hline $70 \%$ & 100.0 & 97.2 & 108.4 & 122.4 & 134.3 \\
\hline $80 \%$ & 100.0 & 96.4 & 106.0 & 118.2 & 127.9 \\
\hline $90 \%$ & 100.0 & 95.4 & 102.7 & 112.7 & 119.3 \\
\hline $10 \%+$ & 100.0 & 93.0 & 99.3 & 104.9 & 109.0 \\
\hline Total & 100.0 & 94.8 & 102.9 & 113.4 & 122.1 \\
\hline \multicolumn{6}{|c|}{ Indicadores de desigualdade } \\
\hline Gini & 0.594 & 0.582 & 0.571 & 0.557 & 0.546 \\
\hline T-Theil & 0.724 & 0.685 & 0.665 & 0.631 & 0.608 \\
\hline \multicolumn{6}{|c|}{ Distribuição relativa da massa de rendimento } \\
\hline $10 \%-$ & 0.4 & 0.5 & 0.7 & 0.7 & 0.6 \\
\hline $20 \%$ & 2.2 & 2.4 & 2.6 & 2.6 & 2.9 \\
\hline $30 \%$ & 3.1 & 3.3 & 3.6 & 3.6 & 3.8 \\
\hline $40 \%$ & 4.4 & 4.6 & 4.9 & 4.9 & 5.4 \\
\hline $50 \%$ & 5.2 & 5.9 & 6.2 & 6.2 & 6.8 \\
\hline $60 \%$ & 5.9 & 5.8 & 5.6 & 5.6 & 6.1 \\
\hline
\end{tabular}

\footnotetext{
${ }^{4}$ De acordo com a PNAD de 2009, a renda média do conjunto das famílias brasileiras apresentava a seguinte composição segundo origem do rendimento: $72,7 \%$ dos rendimentos do trabalho, $27.5 \%$ da proteção social e 3,8\% de juros, aluguéis e transferência de renda (Programa Bolsa Família).

${ }^{5}$ Sobre o tema das desigualdades socioeconômicas, ver Dedecca (2012).
} 


\begin{tabular}{|l|c|c|c|c|c|}
\hline $70 \%$ & 8.7 & 8.3 & 8.5 & 8.5 & 9.0 \\
\hline $80 \%$ & 10.4 & 10.9 & 10.9 & 10.9 & 11.2 \\
\hline $90 \%$ & 16.9 & 16.6 & 16.2 & 16.2 & 15.4 \\
\hline $10 \%+$ & 42.7 & 41.7 & 40.7 & 40.7 & 38.9 \\
\hline Total & 100.0 & 100.0 & 100.0 & 100.0 & 100.0 \\
\hline
\end{tabular}

Fonte: Pesquisa Nacional por Amostra de Domcílios, PNAD/IBGE. Microdados. Deflator: INPC. Elaboração própria.

O reconhecimento dos limites das análises sobre a desigualdade não minimiza nem reduz a importância dos esforços realizados. Ao contrário, explicita, de um lado, a consistência das alterações ocorridas na distribuição da renda corrente, na medida em que a totalidade dos ensaios reconhece a redução da desigualdade nesta dimensão. De outro, qualifica adequadamente a necessidade de se buscar superar tais limites com o objetivo de entender e conhecer a dinâmica das desigualdades socioeconômicas no Brasil, de modo a informar e contribuir para o desenvolvimento dos instrumentos de políticas públicas econômicas e social, com vistas à redução das suas diversas formas de manifestação.

Pode ser tomada como exemplo de adoção dessa perspectiva mais abrangente a nova abordagem estabelecida pelo Governo Federal para sua política de combate à pobreza, o Programa Brasil Sem Miséria. Nessa versão, a política explicita que a renda corrente se constitui em um dos riscos sociais com que se defronta a população pobre, mas também explicita a necessidade de equacionar o acesso dessa população aos bens e serviços públicos como educação, saúde, terra e habitação. A atual definição de pobreza do Governo Federal considera que a situação de fragilidade socioeconômica é de natureza multidimensional, bem como a necessidade de articulação da transferência de renda corrente precisa ser articulada com o provimento adequado de bens e serviços públicos sociais. Em outras palavras, o Programa Brasil Sem Miséria reconhece a existência de um desequilíbrio na distribuição dos bens e serviços públicos sociais, que tem como uma de suas consequências a reprodução da situação de extrema pobreza de uma parcela importante da população brasileira. A iniciativa do Governo Federal converge com abordagens recentes realizadas pelas instituições multilaterais para o desenvolvimento, que tem progressivamente assumido a natureza multidimensional da pobreza e a necessidade de modificação e ampliação do escopo dos programas sociais orientados para sua superação (OECD, 2009b; PNUD, 2010).

Este ensaio não tem o objetivo de desenvolver uma avaliação multidimensional da desigualdade. As observações feitas são importantes para explicitar e reconhecer qual é o limite metodológico e analítico que ela carrega. Adotando a renda corrente como referência para a análise, pretende-se apresentar informação relevante quanto à participação do $1 \%$ tope na sua distribuição durante a década passada. 


\section{A PARTICIPAÇÃO DO 1\% DE DOMICÍLIOS DE MAIOR RENDA NA DISTRIBUIÇÃO DE RENDA CORRENTE}

Como apontado anteriormente, existe ampla convergência sobre a tendência de redução da desigualdade de renda corrente no país na década passada. Esse movimento é confirmado pelos dados do Censo Demográfico 2010. Após um incremento expressivo da desigualdade na década de 1990, associado ao processo inflacionário explosivo com ausência de crescimento no primeiro quinquênio e ao processo de estabilização no segundo, o país conhece uma redução importante durante a década passada. Apesar de ter atingido um patamar inferior ao observado em 1980, quando se encerrou o intenso período de crescimento do após-1964, nota-se que o país continua atualmente a apresentar o indicador mais elevado dentre os países com informação disponível no Relatório de Desenvolvimento Humano do PNUD (2012).

Gráfico 1: Índice de Gini do Rendimento Domiciliar

Per Capita, Brasil, 1991-2000-2010

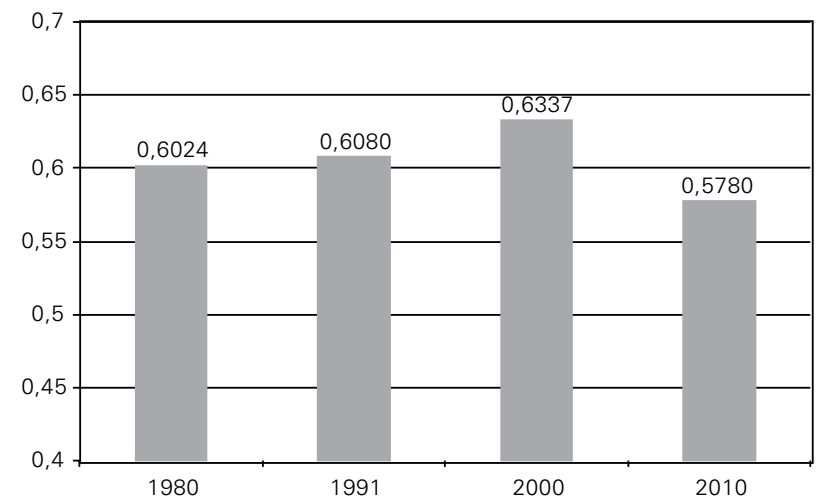

Fonte: Censo Demográfico, IBGE, 1991-2000-2010. Microdados.

Elaboração do autor.

(1) Exclusive os domicílios com rendimento igual a zero.

Tanto os resultados propiciados pela PNAD como pelo Censo Demográfico atestam a tendência de queda da desigualdade da distribuição de renda corrente, que segundo a primeira fonte esteve associada à recuperação generalizada dos rendimentos dos diversos estratos. Considerando esse movimento, este ensaio procura responder à questão seguinte: como evoluiu a participação do $1 \%$ de domicílios de maior renda em uma trajetória de declínio da desigualdade da distribuição de renda corrente com tendência de recuperação dos níveis de rendimentos? ${ }^{6}$

\footnotetext{
${ }^{6}$ Um retrato recente da participação do $1 \%$ tope no Brasil foi realizado por Medeiros (2005), que considerou como ricos os domicílios pertencentes ao estrato. Elaborado a partir dos dados para um período de aumento da desigualdade de renda corrente, o autor explora a elevada participação daqueles domićlíios que denominou como ricos. No esforço atual, não se adota denominação pelos motivos
} 


\section{Por que não chamar o 1\% tope dos domicílios como o $1 \%$ dos domicílios mais ricos?}

Com certeza, esta pergunta será feita por um leitor ao longo do ensaio. A resposta tem referência no debate internacional sobre renda, em especial nas orientações da OIT mencionadas anteriormente.

Como explorado no Quadro 1, os dados que dão sustentação à análise referem-se somente a uma dimensão da renda monetária e não monetária auferida pelos domicílios, sendo que parte preponderante da renda corrente tem origem no trabalho e na proteção social.

Como analisa a literatura internacional, os riscos devem fazer parte do $1 \%$ tope, porém nem todos os domicílios do $1 \%$ tope devem ser considerados ricos. Pois, esta condição está fundamentalmente determinada pela dimensão do patrimônio detido, cuja renda real ou potencial não é expressa somente pela renda do trabalho ou da proteção social e, portanto, pela renda corrente auferida pelos domicílios. Uma parte dos domicílios $1 \%$ tope deve depender exclusiva ou preponderantemente da renda corrente e outra parte deve ter nela uma baixa dependência.

O único país que faz uma avaliação abrangente da desigualdade a partir da renda oriunda do trabalho, da proteção social e do patrimônio, de modo a elucidar a questão anterior, são os Estados Unidos, através do levantamento Survey Consumer Finances produzido pelo Federal Reserve. De acordo com esse levantamento, a renda oriunda da propriedade de capital do primeiro décimo era 20,4 mil vez superior à do primeiro décimo em 2007, enquanto essa relação era de 80 vezes para a renda do trabalho e de 3 vezes para a renda da proteção social.

Estando os dados brasileiros restritos à renda corrente, oriunda fundamentalmente do trabalho e da proteção social, parece ser justificado denominar o $1 \%$ dos domicílios como aqueles de maior renda, pois é provável que parte, mas não a sua totalidade, pertença ao segmento dos ricos brasileiros, os quais têm sua condição desconhecida pelas estatísticas nacionais. 
Tabela 2: Indicadores de Rendimento Domiciliar Per Capita, Brasil, 1991-2000-2010

\begin{tabular}{|c|ccc|ccc|cc|ccc|c|c|}
\hline \multicolumn{7}{|c|}{ Rendimento Médio Per Capita } & \multicolumn{4}{c|}{ Massa de rendimentos } \\
\hline \multicolumn{2}{|c|}{ Em Reais de 2010 } & \multicolumn{3}{|c|}{ Relativo } & \multicolumn{3}{c|}{$\%$ a.a. } & \multicolumn{3}{c|}{ Relativo } & \multicolumn{2}{c|}{$\%$ a.a. } \\
\hline $10 \%-$ & 45 & 49 & 78 & 1.0 & 1.0 & 1.0 & 1.1 & 4.7 & 0.7 & 0.6 & 0.8 & 2.0 & 9.0 \\
$20 \%$ & 89 & 103 & 181 & 2.0 & 2.1 & 2.3 & 1.6 & 5.8 & 1.3 & 1.2 & 1.8 & 3.5 & 9.5 \\
$30 \%$ & 135 & 153 & 265 & 3.0 & 3.1 & 3.4 & 1.4 & 5.6 & 2.2 & 1.9 & 2.7 & 3.1 & 8.8 \\
$40 \%$ & 191 & 210 & 355 & 4.3 & 4.3 & 4.6 & 1.1 & 5.4 & 2.9 & 2.6 & 3.6 & 3.4 & 8.5 \\
$50 \%$ & 252 & 281 & 460 & 5.6 & 5.7 & 5.9 & 1.2 & 5.0 & 3.7 & 4.0 & 3.8 & 5.4 & 4.8 \\
$60 \%$ & 340 & 352 & 539 & 7.6 & 7.1 & 6.9 & 0.4 & 4.3 & 5.1 & 4.4 & 6.4 & 2.7 & 9.3 \\
$70 \%$ & 466 & 470 & 706 & 10.4 & 9.5 & 9.1 & 0.1 & 4.1 & 7.2 & 6.3 & 7.4 & 3.0 & 6.9 \\
$80 \%$ & 665 & 665 & 950 & 14.9 & 13.5 & 12.2 & 0.0 & 3.6 & 10.1 & 9.3 & 9.2 & 3.6 & 5.1 \\
$90 \%$ & 1,067 & 1,087 & 1,444 & 23.8 & 22.0 & 18.5 & 0.2 & 2.9 & 16.2 & 15.7 & 14.5 & 4.1 & 4.4 \\
$5 \%+$ & 4,877 & 5,486 & 7,463 & 109.0 & 111.1 & 95.9 & 1.3 & 3.1 & 36.9 & 40.5 & 37.5 & 5.6 & 4.4 \\
$1 \%+$ & 10,541 & 12,177 & 17,868 & 235.6 & 246.6 & 229.5 & 1.6 & 3.9 & 16.2 & 18.3 & 18.2 & 6.0 & 5.1 \\
Total & 658 & 763 & 993 & 14.7 & 15.5 & 12.8 & 1.7 & 2.7 & 100.0 & 100.0 & 100.0 & 4.5 & 5.2 \\
\hline
\end{tabular}

Fonte: Censo Demográfico, IBGE, 1991-2000-2010. Microdados. Elaboração do autor.

$\mathrm{Na}$ Tabela 2 encontra-se a evolução dos rendimentos correntes segundo estratos de renda domiciliar per capita entre 1991 e 2010. O primeiro aspecto a se destacar do movimento refere-se às diferenças de ganhos de renda segundo os diversos estratos nas duas décadas. Enquanto na década de 1990 o último percentil superior teve um ganho de renda mais expressivo que os observados para todos os demais estratos, constata-se que, na primeira década deste século, $70 \%$ dos domicílios de menor renda tiveram ganhos maiores que os encontrados para os $30 \%$ superiores, inclusive ao auferido pelo $1 \%$ de domićlios de renda mais elevada ${ }^{7}$. Isto é, a recuperação de renda foi mais intensa para os estratos inferiores, devendo-se, ainda, ressaltar que o movimento se propagou na estrutura de rendimento como um todo. Ademais, os ganhos de renda auferidos pelos estratos inferiores na década passada foram significativamente maiores que os encontrados no período anterior, dando evidências da importância do crescimento com políticas públicas adequadas, seja para a redução da desigualdade, seja para uma apropriação mais justa dos frutos da expansão pelos domicílios mais pobres.

Este resultado explicita imédiatamente que a recuperação atual da economia e as instituições públicas que a sustentam têm produzido uma recuperação da renda corrente que favorece relativamente mais os de menor renda, mas que também tem beneficiado os estratos superiores da distribuição. De um lado, o processo tem se traduzido em um movimento de elevação do bem-estar potencial relacionado à renda corrente dos domicílios, independentemente do estrato a que pertençam. Por

\footnotetext{
${ }^{7}$ Para a análise da distribuição de renda corrente se considerou somente as famílias com rendimento maior que zero, não tendo sido aplicada restrição de limite superior de renda. Hoffmann et al. (2008) adotaram o limite superior de $\mathrm{R} \$ 30.000,00$ para o ano de 2000 , chegando a uma participação do $1 \%$ tope de $16 \%$, patamar um pouco inferior ao observado por este estudo.
} 
outro, ele explica a modificação tênue da participação dos diversos estratos na massa total de renda corrente.

De acordo com a Tabela 2 , os $10 \%$ de domicílios de menor renda se apropriavam, em 2010 , de $0,8 \%$ da massa de rendimentos, enquanto o $1 \%$ de domicílios do último estrato respondia por $18,2 \%$. Isto é, a redução da desigualdade não implicou a perda de participação dos domicílios $1 \%$ tope. Se essa situação não reitera conclusões encontradas na literatura internacional, de aumento da participação desse estrato, também mostra que o padrão de crescimento foi, ao menos até 2010 , insuficiente para levar que ele produzisse uma alteração do perfil da massa de rendimentos, que pudesse se traduzir em um movimento de maior homogeneização da estrutura socioeconômica brasileira em termos de renda corrente.

É interessante observar essa capacidade de preservação da participação de $1 \%$ de domicílios de maior renda em uma perspectiva regional. Como mostra a Tabela 2, o crescimento fortaleceu a renda dos domicílios da região Sudeste em relação à média nacional, bem como foi seguido da preservação do rendimento dos domicílios da região Norte. Esta tendência foi destoante daquela observada para o conjunto dos domicílios, que conheceu uma redução da diferença dos rendimentos regionais em relação à média. Mesmo assim, é importante ressaltar que a redução da desigualdade para o conjunto dos domicílios foi mais intensa para a região Sul, seguida daquelas das regiões Sudeste, Nordeste, Centro-Oeste e Norte (Anexo 1).

Quando analisada a distribuição de domicílios, nota-se que o 1\% de domićlios de maior renda das regiões Norte e Sudeste teve aumento superior ao observado para o conjunto dos domicílios. Ademais, percebe-se que a reprodução dos domicílios do $1 \%$ tope tem sido relativamente mais expressiva nas regiões de menor renda. Há, portanto, uma redistribuição do $1 \%$ dos domicílios de maior renda para as regiões de menor desenvolvimento, movimento que pode conter uma possível diminuição da heterogeneidade regional entre os domicílios de maior renda e que também deve contribuir para um recuo menos intenso da desigualdade média entre as regiões.

Tabela 3: Indicadores Relativos de Rendimento Domiciliar Per Capita e de Distribuição de Domicílios segundo Região Geográfica, Brasil, 2000-2010

\begin{tabular}{|c|c|c|c|c|c|c|c|c|c|c|}
\hline \multicolumn{7}{|c|}{ Rendimento Per Capita } & \multicolumn{4}{|c|}{ Domicílios } \\
\hline & \multicolumn{2}{|c|}{$\%$ a.a. } & \multicolumn{2}{|c|}{$1 \%+$} & \multicolumn{2}{|c|}{ Total } & \multicolumn{2}{|c|}{$1 \%+$} & \multicolumn{2}{|c|}{ Total } \\
\hline & 99 & Total & 2000 & 2010 & 2000 & 2010 & 2000 & 2010 & 2000 & 2010 \\
\hline Norte & 4.0 & 3.4 & 106.1 & 106.8 & 64.2 & 69.0 & 2.8 & 3.4 & 6.1 & 6.7 \\
\hline Nordeste & 3.6 & 3.9 & 99.8 & 96.7 & 53.7 & 60.4 & 9.1 & 12.2 & 24.7 & 25.9 \\
\hline Sudeste & 4.3 & 2.2 & 98.7 & 102.4 & 126.0 & 120.9 & 63.7 & 58.3 & 45.6 & 44.0 \\
\hline Sul & 3.4 & 3.1 & 100.9 & 96.3 & 107.3 & 111.7 & 15.6 & 14.9 & 16.4 & 15.8 \\
\hline Centro-Oeste & 2.6 & 3.5 & 106.5 & 94.1 & 108.1 & 117.1 & 8.8 & 11.2 & 7.1 & 7.6 \\
\hline Total & 3.9 & 2.7 & 100.0 & 100.0 & 100.0 & 100.0 & 100.0 & 100.0 & 100.0 & 100.0 \\
\hline
\end{tabular}

Fonte: Censo Demográfico, IBGE, 2000-2010. Microdados. Elaboração do autor.

Explorada a tendência de evolução dos rendimentos segundo os estratos, com foco naquele do $1 \%$ de domicílios de maior renda, é interessante completar a análise com as informações correspondentes sobre participação e atividade econômica 
dos diversos estratos. Segundo a Tabela 4, a participação econômica potencial, expressa na relação população em idade ativa e população total, aumentou para todos os estratos de renda entre 2000 e 2010, sendo que o último percentil apresenta um patamar significativamente superior ao encontrado para os estratos inferiores.

Quanto à participação econômica efetiva, conhecida pela relação população econômica ativa sobre população em idade ativa, nota-se um padrão semelhante. Os domicílios de maior renda tendem a ter uma taxa de participação mais elevada, comparativamente à encontrada para aqueles dos estratos inferiores. A menor participação nesses estratos é ainda acompanhada de uma taxa de desemprego significativamente mais elevada, bem como de uma maior participação da ocupação agrícola (POA) na ocupação total (PO) mais expressiva. Enquanto a taxa de desemprego no último percentil era de 1,2\% em 2010, representando metade daquela observada no início da década, ela correspondia a $19,6 \%$ para o primeiro décimo inferior, tendo este estrato conhecido uma redução de somente $26 \%$ ao longo do período. Em termos relativos, a taxa de desemprego do primeiro décimo inferior era 16 vezes mais elevada que a observada para o 1\% tope em 2010.

Tabela 4: Taxas de Atividade, Participação, Desemprego e Ocupação segundo Estratos de Renda Domiciliar Per Capita, Brasil, 2000-2010

\begin{tabular}{|c|cc|cc|cc|cc|}
\hline \multicolumn{7}{|c|}{ Taxas de Atividade, Participação, Desemprego e Ocupação } \\
\hline & \multicolumn{3}{|c|}{2000} & \multicolumn{3}{c|}{2010} \\
\hline & PIA/PT & PEA/PIA & PD/PEA & POA/PO & PIA/PT & PEA/PIA & PD/PEA & POA/PO \\
\hline $10 \%-$ & 64.8 & 48.2 & 23.3 & 44.3 & 73.2 & 38.8 & 19.6 & 47.9 \\
\hline $20 \%$ & 70.7 & 51.3 & 21.9 & 30.8 & 77.5 & 47.3 & 14.0 & 23.7 \\
\hline $30 \%$ & 75.6 & 52.5 & 20.2 & 23.5 & 82.4 & 51.5 & 11.2 & 16.9 \\
\hline $40 \%$ & 77.5 & 57.2 & 17.0 & 18.2 & 84.5 & 57.7 & 8.1 & 12.7 \\
\hline $50 \%$ & 82.1 & 53.3 & 14.9 & 15.5 & 86.2 & 63.5 & 6.1 & 9.5 \\
\hline $60 \%$ & 81.0 & 62.5 & 13.3 & 10.8 & 91.9 & 57.1 & 4.3 & 10.5 \\
\hline $70 \%$ & 84.4 & 64.2 & 11.2 & 8.1 & 90.2 & 68.9 & 3.8 & 6.9 \\
\hline $80 \%$ & 86.1 & 64.6 & 9.6 & 5.4 & 91.6 & 69.5 & 3.2 & 5.7 \\
\hline $90 \%$ & 87.5 & 64.6 & 7.9 & 3.4 & 92.0 & 70.6 & 2.8 & 4.1 \\
\hline $5 \%+$ & 88.7 & 64.3 & 6.1 & 2.5 & 92.5 & 70.4 & 2.3 & 3.0 \\
\hline $1 \%+$ & 92.9 & 70.5 & 2.3 & 1.7 & 94.5 & 76.1 & 1.2 & 2.4 \\
\hline Total & 79.1 & 58.4 & 13.9 & 14.3 & 84.9 & 58.5 & 7.1 & 12.5 \\
\hline
\end{tabular}

Fonte: Censo Demográfico, IBGE, 1991-2000-2010. Microdados. Elaboração do autor.

Os dados de participação e atividade revelam que a recuperação do mercado de trabalho parece ter favorecido relativamente mais os domicílios de maior renda, abrindo-lhes uma vantagem potencial em termos econômicos. A partir dos resultados encontrados nos diversos estudos realizados com os dados da PNAD, e que foram anteriormente mencionados, é possível afirmar que a vantagem ocupacional observada para o último percentil, também encontrada para os décimos superiores, não deve ter se efetivado em termos de renda graças às políticas públicas de trans- 
ferência de renda e do salário mínimo ${ }^{8}$, que foram decisivas para a evolução dos rendimentos dos estratos inferiores.

Considerada a cor do chefe do domicílio, percebe-se um aumento da participação daqueles de cor negra dentre o $1 \%$ de domicílios de maior renda entre 2000 e 2010, sendo ela mais expressiva nas regiões Norte e Nordeste. Mesmo assim, a participação de chefes negros no $1 \%$ tope continua sendo inferior à encontrada para a média da população brasileira, sendo que a menor expressão é observada para as regiões consideradas de maior desenvolvimento, isto é, Sudeste e Sul. No caso desta última região, onde a participação de chefes negros é ainda mais baixa dentre sua população, sua participação entre as famílias do último percentil apresenta o menor nível absoluto e relativo, quando comparado com a do total dos domićlios. Este resultado indica que quanto menor a presença de chefes negros para o conjunto dos domicílios da região, menor deve ser a probabilidade de um domicilio do último percentil ter um chefe negro na sua condução.

Tabela 5: Participação dos Domicílios com Chefes de Cor Negra no Total dos Domicílios segundo Região Geográfica, Brasil, 2000-2010

\begin{tabular}{|l|cc|cc|}
\hline \multicolumn{4}{|c|}{ Participação dos Domicílios com Chefes de Cor Negra no Total dos Domicílios } \\
\hline & \multicolumn{2}{|c|}{$1 \%+$} & \multicolumn{2}{c|}{ Total } \\
\hline & 2000 & 2010 & 2000 & 2010 \\
\hline Norte & 38.3 & 42.0 & 68.9 & 73.4 \\
\hline Nordeste & 28.2 & 33.7 & 65.6 & 69.1 \\
\hline Sudeste & 5.6 & 9.9 & 35.3 & 42.6 \\
\hline Sul & 2.5 & 4.5 & 15.3 & 20.1 \\
\hline Centro-Oeste & 18.1 & 25.6 & 48.5 & 55.8 \\
\hline Total & 9.2 & 14.9 & 42.5 & 49.0 \\
\hline
\end{tabular}

Fonte: Censo Demográfico, IBGE, 2000-2010. Microdados. Elaboração do autor.

Em relação ao recorte urbano/rural, nota-se que o $1 \%$ tope dos domicílios é predominantemente urbano, sendo que esta característica foi acentuada ao longo da década passada. O recuo da participação dos domicílios do último percentil foi mais intenso que o observado para o conjunto da população, reiterando movimento já analisado quanto à participação da ocupação agrícola9 . Este resultado sugere

\footnotetext{
${ }^{8}$ Em razão da lógica de construção do questionário, não é possível identificar os rendimentos oriundos das políticas públicas segundo as diversas modalidades existentes, como aposentadorias, pensões, transferências de renda (Bolsa Família) etc. Somente a renda do trabalho e a renda total da proteção social podem ser conhecidas na base de microdados, restringindo a análise dos ganhos de renda dos diversos estratos segundo as respectivas formas.

${ }^{9}$ Deve-se ressaltar que parte do $1 \%$ tope dos domicílios urbanos deve ter sua renda oriunda da atividade agrícola. Entretanto, os domicílios nessa situação apresentam maior possibilidade de acesso aos bens públicos. A expressão mais intensa de domicílios rurais nos estratos inferiores aponta menor possibilidade desse acesso, em razão da maior dificuldade de desenvolvimento nessas áreas da infraestrutura de saneamento, saúde, educação, particularmente quando se considera a heterogeneidade da distribuição populacional e da infraestrutura social que carrega o país.
} 
uma tendência de progressivo adensamento dos domicílios de maior renda no espaço urbano, em todas as regiões, notando-se um movimento semelhante e mais lento para o conjunto dos domicílios brasileiros. Do ponto de vista do recorte rural/ urbano, percebe-se a maior probabilidade de um domicílio rural pertencer aos estratos inferiores e de um urbano participar dos estratos superiores. Neste sentido, a perspectiva de recorrência de uma população ao redor de 30 milhões de pessoas no meio rural, ao menos até 2030 , indica a necessidade de a política pública atuar com o objetivo de impedir que tal contingente expressivo de brasileiros fique progressivamente concentrado nos estratos mais baixos da distribuição.

Tabela 6: Participação dos Domicílios Rurais no Total de

Domicílios segundo Região Geográfica, Brasil, 2000-2010

\begin{tabular}{|l|cc|cc|}
\hline & \multicolumn{2}{|c|}{$1 \%+$} & \multicolumn{2}{c|}{ Total } \\
\hline & 2000 & 2010 & 2000 & 2010 \\
\hline Norte & 6.6 & 4.4 & 25.9 & 22.5 \\
\hline Nordeste & 2.8 & 1.5 & 27.1 & 24.5 \\
\hline Sudeste & 1.7 & 1.1 & 8.4 & 6.6 \\
\hline Sul & 4.6 & 4.5 & 17.7 & 14.2 \\
\hline Centro-Oeste & 4.8 & 2.4 & 12.8 & 10.6 \\
\hline Total & 2.7 & 1.9 & 15.9 & 13.8 \\
\hline
\end{tabular}

Fonte: Censo Demográfico, IBGE, 2000-2010. Microdados. Elaboração do autor.

\section{ALGUMAS OBSERVAÇÕES FINAIS}

A análise da redução da desigualdade de renda corrente se fez com a perda de participação dos estratos intermediários. Os resultados apresentados mostram uma renitência da participação do $1 \%$ tope na distribuição de renda corrente do país, bem como de sua condição de inserção econômica mais favorável, em especial quando comparada com os primeiros décimos. Existe uma grande expectativa de que o movimento de redução da desigualdade econômica tenha continuidade nesta década, porém é fundamental que ele seja caracterizado por uma diminuição da participação do $1 \%$ tope de domicílios, a qual é facilmente justificada pela discrepância entre sua participação na massa total de rendimento corrente e a dos $10 \%$ dos domicílios de menor renda.

Ademais, esse argumento ganha respaldo quando se compara a participação do $1 \%$ tope no Brasil com os países da OCDE (Tabela 7). No final da década passada, nossa situação era comparada à dos Estados Unidos, a qual tem sido internacionalmente reconhecida como problemática e apontada como um entrave ao processo de retomada sustentada da economia daquele país no pós-crise de 2009 (CBO, 2011; Atkinson et al., 2011; Mishell, 2012; Noah, 2012).

Tendo como perspectiva ser a quarta ou quinta economia do mundo no final desta década, objetivo em geral associado a um desenvolvimento com maior equidade social, é fundamental que se reduza a participação do $1 \%$ tope na massa de rendimento corrente, bem como que sejam melhoradas as condições de acesso ao mercado de trabalho e aos bens e serviços públicos dos estratos inferiores de renda. 
Tal alteração é decisiva também para sustentar um ciclo de crescimento assentado nos mercados de bens e serviços de consumo e sociais, dado que são os estratos inferiores os mais carentes em termos de acesso a esses bens.

Este objetivo tem relevância se considerada a avaliação da OCDE quanto ao fato de o Brasil ter destoado em termos de comportamento da desigualdade de renda corrente na década passada. Para reiterarmos tal resultado é preciso que o $1 \%$ tope ceda participação aos demais estratos de renda, permitindo reduzir a desigualdade, de modo a fortalecer a renda dos estratos inferiores e intermediários, processo fundamental para ampliar o mercado interno e dar viabilidade a um crescimento sustentado que se traduza em desenvolvimento com maior justiça social.

Tabela 7: Participação do $1 \%$ tope de domicílios na massa total de rendimento corrente domiciliar, Países Selecionados, 1991-2000-2008

\begin{tabular}{|l|c|c|c|}
\hline & \multicolumn{3}{|c|}{ Em Porcentagem } \\
\hline & 1991 & 2000 & $2008\left(^{*}\right)$ \\
\hline Brasil (2010) & 16.2 & 18.3 & 8.2 \\
\hline Austrália & 6.4 & 7.2 & 13.3 \\
\hline Canadá (2007) & 9.2 & 12.4 & 9.0 \\
\hline Nova Zelândia (2005) & 8.0 & 8.3 & 14.3 \\
\hline Reino Unido (2005) & 10.3 & 12.7 & 17.7 \\
\hline Estados Unidos & 12.2 & 16.5 & 8.9 \\
\hline França (2006) & 8.0 & 8.3 & 9.2 \\
\hline Japão (2005) & 7.5 & 8.2 & 9.0 \\
\hline Itália (2004) & 7.8 & 9.1 & 7.5 \\
\hline Noruega & 4.5 & 8.3 & 9.8 \\
\hline Portugal (2005) & 7.5 & 9.1 & 8.8 \\
\hline Espanha (2005) & 8.1 & 8.8 & 7.1 \\
\hline Suécia & 5.1 & 6.0 & \\
\hline
\end{tabular}

Fontes: OCDE, Divided We Stand: Why Inequality Keeps Rising, Paris, 2011; Censo Demográfico, IBGE, 1991-2000-2010. Elaboração do autor.

(*) Quando não especificado, a informação refere-se ao ano de 2008.

$* * * * *$

Anexo 1

Índice de Gini da Renda Domiciliar Per Capita segundo Região Geográfica, Brasil, 2000-2010

\begin{tabular}{|c|cc|cc|c|}
\hline \multirow{2}{*}{ Norte } & \multicolumn{2}{|c|}{ Absoluto } & \multicolumn{2}{c|}{ Relativo } & Variação Absoluta \\
\cline { 2 - 6 } & 2000 & 2010 & 2000 & 2010 & $2010-2000$ \\
\hline Nordeste & 0.6312 & 0.6027 & 100 & 104 & -0.0285 \\
\hline Sudeste & 0.6402 & 0.5863 & 101 & 101 & -0.0539 \\
\hline Sul & 0.5881 & 0.5132 & 98 & 97 & -0.0582 \\
\hline Centro-Oeste & 0.6043 & 0.5637 & 93 & 89 & -0.0749 \\
\hline Total & 0.6337 & 0.5780 & 100 & 98 & -0.0406 \\
\hline
\end{tabular}

Fonte: Censo Demográfico, IBGE, 1991-2000-2010. Microdados. Elaboração do autor. 


\section{REFERÊNCIAS BIBLIOGRÁFICAS}

APSA - American Political Science Associaton (2004) "American Democracy in an Age of Rising Inequality”, Washington DC: American Political Science Association. Documento disponível no endereço http://www.apsanet.org/imgtest/taskforcereport.pdf.

Atkinson, A. \& T. Piketty (eds.) (2009) Top Incomes over the Twentieth Century: A Contrast Between Continental European and English Speaking Countries, Oxford: Oxford University Press.

Atkinson, A.B. \& J.E. Søgaard, (2013) "The long-run history of income inequality in Denmark: Top incomes from 1870 to 2010", EPRU Working Paper Series, n. 1, Copenhagen: Economic Policy Research Unit/ Department of Economics/ University of Copenhagen.

Atkinson, A. \& S. Morelli (2011) "Inequality and Banking Crises: a first look”, Global Labour Forum, mimeo, Turin: OIT.

Barros, R.P.; Carvalho, M.; Mendonça, R.; Franco, S. (2010) “Determinantes da queda de desigualdade de renda no Brasil, Série Seminários, Brasília: IPEA.

Barros, R.P; Foguel, M.N.; Ulyssea, G. (2007) Desigualdade de renda no Brasil: uma análise da queda recente, Volume 1, Brasília: IPEA.

Barros, R.P; Foguel, M.N.; Ulyssea, G. (2008) Desigualdade de renda no Brasil: uma análise da queda recente, Volume 2, Brasília: IPEA.

Bihr, A. \& R. Pfefferkorn (2008) Le système des inégalités, Paris: La Découverte.

Cacciamali, M.C. (2011) "Brasil: un caso reciente de crecimiento económico con distribución de renta", Revista de Estudios Empresariales, 1, Madrid: Segunda Época.

CBO - Congressional Budget Office / Congress of the United States (2011) Trends in the Distribution of Household Income Between 1979 and 2007, Washington DC: Congress Of United States.

Dedecca, C.S. (2007) “A redução da desigualdade no Brasil, uma estratégia complexa”, in Barros, R.P; Foguel, M.N.; Ulyssea, G. (2007).

Dedecca, C.S. (2012) “Desigualdade, mas de qual falamos?”, Revista de Economia Política, 32(1), São Paulo: Editora 34.

Dell, F. (2007) “Top Incomes in Germany Throughout the Twentieth Century: 1891-1998”, in A. Atkinson and T. Piketty (eds.), Top Incomes over the Twentieth Century: A Contrast Between Continental European and English Speaking Countries. Oxford: Oxford University Press.

DESA/UN (2005) Inequality Predicament, New York: United Nation.

Gadrey, J. \& F. Jany-Catrice (2003) Les indicateurs de richesse et de développement, un bilan international en vue d'une inciative française, Rapport Dares, Paris: Ministère du Travail et de la Solidarité.

Gustafsson, B. \& B. Jansson (2007) “Top Incomes in Sweden during Three-Quarters of a Century: A Micro Data Approach", IZA Discussion Paper 2672. Institute for the Study of Labor, Bonn.

Hicks, J.R. (1940) “The Valuation of the Social Income”, Economica, New Series, Vol. 7, No. 26, May, London: Blackwell Publishing/London School of Economics and Political Science.

Hicks, J.R. (1948) “The Valuation of the Social Income-a Comment on Professor Kuznets' Reflections", Economica, New Series, Vol. 15, No. 59, Aug, London: Blackwell Publishing/London School of Economics and Political Science.

Hoffmann, R. (2007) “Queda da Desigualdade da Distribuição de Renda no Brasil, de 1995 a 2005”, in Barros, R.P; Foguel, M.N.; Ulyssea, G. (2007).

Hoffmann, R. \& M.G. Ney, (2008) A recente queda da desigualdade de renda no Brasil: análise de dados da PNAD, do Censo Demográfico e das Contas Nacionais, Econômica, 10(1), Niterói: UFF.

ILO - International Labour Organization (1998) Measurement of income from employment, Report II, Sixteenth International Conference of Labour Statisticians, Geneva: International Labour Organization.

ILO - International Labour Organization, (2003) Household income and expenditure Statistics, Seventeenth International Conference of Labour Statisticians, Geneva: International Labour Organization.

Kertenetzky, C. L. (2009) “Redistribuição e Desenvolvimento? A Economia Política do Programa Bolsa Família”, Dados, 52, Rio de Janeiro: Anpocs. 
Kuznets, S. (1973) "Remarks", in M.Moss, The Measurement of Economic and Social Performance, New York: National Bureau of Economics Research/Columbia University Press.

Landais, C. (2007) “Les hauts revenus en France(1998-2006): Une explosion des inégalités?”, mimeo, Paris: Paris School of Economics.

Lavinas, L. (2010) "Pobreza: Métricas e Evolução Recente no Brasil e no Nordeste”, Cadernos do Desenvolvimento, 5, Rio de Janeiro: Centro Celso Furtado.

Leigh, A. (2009) “Top Incomes," in W. Salverda, B. Nolan e T. Smeeding (Eds.), The Oxford Handbook of Economic Inequality, Oxford: Oxford University Press.

Mishel, L. \& N. Sabadish (2012) "CEO pay and the top 1\%”, Issue Brief, 331, Washington DC: Economic Policy Institute.

Moriguchi, C. \& E. Saez (2007) “The Evolution of Top Wage Incomes in Japan, 1951-2005”, mimeo, Northwestern University.

Nolan, B. (2007) "Long-Term Trends in Top Income Shares in Ireland". in A. Atkinson and T. Piketty (Eds.), Top Incomes over the Twentieth Century: A Contrast Between Continental European and English Speaking Countries. Oxford: Oxford University Press.

OECD (2008) Growing Unequal? Income distribution and poverty in the OECD countries, Paris: OECD.

OECD (2009) Protection sociale, lutte contre la pauvreté et croissance pro-pauvres, Note d'orientation, Paris: Oecd.

OECD (2011) Divided we stand, why inequality keeps rising, Paris: OECD.

Oliveira, A. \& E. Rios Neto, (2006) "Tendências da desigualdade salarial para coortes de mulheres brancas e negras no Brasil", Estudos Econômicos, 36 (2): 205-236.

Osberg, L. \& A. Sharpe, (2003) "New Estimates of the Index of Economic Well-being for Selected OECD Countries", mimeo, Toronto: Center for the Study of Living Standards.

Medeiros, M. (2005) “O estudo dos ricos no Brasil”, Econômica, 7(1), Niterói: UFF.

Piketty, T. \& E. Saez (2006) "The Evolution of Top Incomes: a Historical and International Perspective", American Economic Review, vol. 96(2).

Programa das Nações Unidas para o Desenvolvimento, PNUD (2010) Relatório de Desenvolvimento Humano, Washington DC: Nações Unidas.

Saboia, J.M. (2010) "Elasticidades dos Rendimentos do Trabalho em Relação ao Salário Mínimo - A Experiência do Período Recente", Economia e Sociedade, 19, Campinas: Unicamp.

Salm, C. (2007) "Sobre a recente queda da desigualdade de renda no Brasil: uma leitura crítica", in Barros, R.P; Foguel, M.N.; Ulyssea, G. (2007).

Sen, A. (1992) Inequality reexamined, New York: Russel Sage Foundation/Clarendon Press.

Soares, S.S.D. (2011) "A Desigualdade de Renda de 1995 a 2009 e Tendências Recentes", Texto para Discussão, Niterói: Centro de Estudos de Desenvolvimento e Desigualdade/UFF.

Noah, T. (2012) The Great Divergence: America's Growing Inequality Crisis and What We Can Do about It, New York: Bloomsbury Press.

Weinberg, D.H. (2004) Income Data Quality Issues in US Census Bureau, The Annual Social and Economic Supplement to the Current Population Survey, Washington DC: US Census Bureau.

Weinberg, D.H. (2006) "Income data quality issues in the CPS," Monthly Labor Review, June, Washington DC: Bureau of Labor Statistics.

Wolff, E.; Zacharias, A.; Masterson, T. (2009) "Long-term tends in the levy institute of measurement of economic well-being (LIMEW), United States, 1959-2004”, Working Paper 556, Annandale-on-Hudson: The Levy Economics Institute.

Zacharias, A. (2002) “A Note on the Hicksian Concept of Income”, Levy Institut of Bard College Working Paper, 342, Annandale-on-Hudson: Levy Economics Institute. 